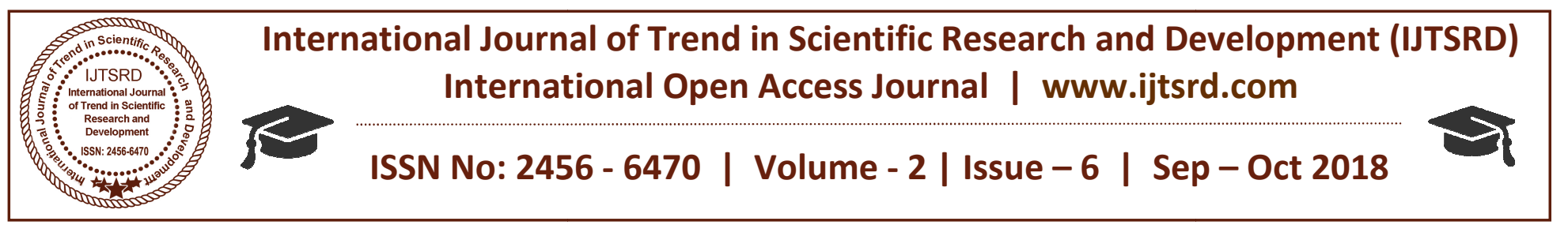

\title{
Foray Spotlight System
}

\author{
Mrs A. Nalini \\ Lecturer (Senior Grade), Department of Computer Engineering, \\ Nachimuthu Polytechnic College, Pollachi, Tamil Nadu, India
}

\begin{abstract}
This Project "Foray spotlight system" is used for protecting the Computer from Malware attacks. It is a real time project and system security software which is used to identify the malicious activities in a computer. Malwares are the main problem for computers. Once it enters into the computer it will corrupt the files. Foray spotlight system acts like an antivirus software. It detects malwares like other antivirus software's, but it has some more additional advantages. Malwares are easily spread over the computer at the time of connecting the external devices like Pen-drive, Card reader, etc. and it has various steps to clear malwares. First it checks for a malware and asks the user whether to destroy or not. If the user chooses destroy option it will destroy file or else choose track option for tracking for malware. If it is able to track, then it will clear the malware. If it is unable to track, it will block the file. It has more graphical interface than the antivirus software's. Additional feature like auto boost technology increase the performance of the system. It identifies and removes the unwanted running programs. This Malwares detection method is more effective than other system protecting software.
\end{abstract}

Keyword: Malvares, Threads, Pen drive, auto boost

\section{INTRODUCTION}

This project is used to detect and delete the virus in the system .It consists of 3 types of scans. They are customs scan, full scan and quick scan. This also has a special feature that it will automatically free up the memory in a particular drive. All these features are integrated within the project .The memory space required for this project is below 3MB.Spartans antivirus is user-friendly and the Graphical User Interface is also very clear and easy to use. Spartans have big database to identify and detect the various types of virus. Uploading and modifying the virus can be done in Spartans. It has boost up tool that detect the running virus and boost up the performance of the system.

Most important function of any antivirus is virus scan engine. It scans the information and if the viruses are detected, it disinfects them. The information can be scanned in different ways.

Size: it can easy detect if the file is infected or altered. Some viruses append their malicious code at the end of the file. An antivirus scanner (scan engine) scans it and compares it before and after sizes. If there is no modification done by the user so it suspects that there is some malicious activity running.

Pattern Matching: every virus has a unique signature that they use to infect the files or computers. This signature could be some lines in assembly language that overwrite the stack pointer and then jump to the new line of code. An antivirus program compares the information with a virus database (virus signature). If information matches any of the virus signatures then antivirus shows that the file is infected by the virus.

Heuristic: if any information being scanned is dangerous and without knowing that is it contains a virus or not? This method is known as heuristic scanning. It analyse that how an information acting and comparing it with the list of dangerous activities. For e.g. if an antivirus program notices that a program is trying to open every EXE file on your computer and infecting it by writing a copy of the original program into it. So an antivirus program detects this program and declares it a dangerous activity or unknown type of virus and sound the alarm. Then it is up to user weather the danger should be eliminated or not. 
These methods have their pros and cons. If the antivirus program uses virus signature mechanism then it must update it at least once a day because 15 new viruses we discovered every day. If an antivirus left for two or more days without updating it cause a serious danger.

\section{EXISTING SYSTEM}

Antivirus will identify the virus and detect the virus. But it doesn't have a real time protection method. They do not delete the archive files. It doesn't replace the deleted files from our computer. Lot of memory is required for Grafical user interface. Scanning time duration is high. It detects and delets corrupted files. Hidden files can't get back. Some features need to be added for this. History of deleted virus lists are not stored. Antivirus cannot identify the newly created virus.

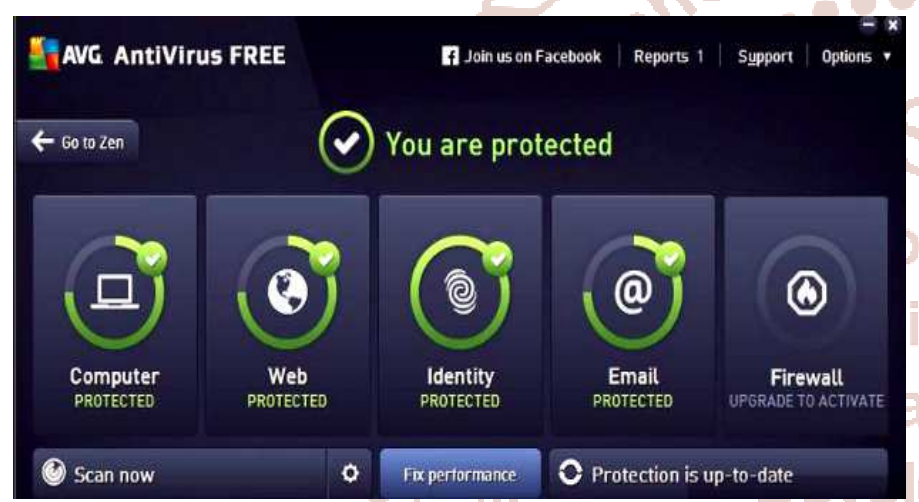

FIG1. ANTIVIRUS MODULE

\section{Types of Worms}

A few different types of worms are emailing worms, instant messaging worms, internet worms and filesharing networks worms. emailing worms are those in the attachments that are sometimes sent with emails. instant messaging worms usually infect a computer when an infected link is sent to a person and they open it. these also get into computers and automatically send to most if not all of the people on your buddy list. internet worms usually scan different computers and try to get into their systems. a lot of times they will try and be downloaded onto the computer by sending a request to be downloaded. filesharing networks worms usually copy itself in a shared file under a name that is not suspicious and will start to infect the computer as well as those in the same network.

\section{PROPOSED SYSTEM}

Spartans antiviruses have the custom scan method for scanning particular drives. It has real time protection method. If any unwanted malicious files is found or installed means, it will alert the user. History of the virus are listed in the registry form. Every user must register their details to create an account. Each user has permission to update a new virus and give suggestions for developing security. It is an open source software, easy to customize with Graphical user interface. Software does not require any framework application and is a simple installation process. The deleted files can be recovered in this project. If any virus enters the system, the antivirus software will report to user and block the virus. Corrupted files could be filtered and stored into drives. It supports all Hardware and software components.

\section{LIST OF MODULES}

Home page module.

Quick scan module.

Full scan module.

\section{Custom scan module.}

\section{HOME PAGE MODULE}

Home page contains scan options like quick scan, full scan, custom scan options. User can select this options to scan the drives. Some settings are provided for the User. History of detected viruses is visible for the users and virus updatation is also available. Additional features available for users are Manage, Tools, Advanced Protection, Real time protection.

\section{QUICK SCAN MODULE}

This module scans the drive very quickly. If any threat is found, it is automatically removed. All the memory files are scanned by this module.

\section{FULL SCAN MODULE}

Full scan method scans smoothly and slowly. It scans the whole system and if any threat is found, it is informed to the user. This method doesn't delete the malicious files immediately.

\section{CUSTOM SCAN MODULE}

Custom scan method is advantage for the user. If user wants to scan any particular drive, it is possible by this method. Custom scan helps to delete the malicious file in particular disks or Removable drives.

\section{FUTURE ENHANCEMENT}

This software has full details about all types of virus and affecting methods of virus. Database has all kind of virus so it can block all type of viruses. If virus 
attacks the system at any time, real time protection security will scans automatically and delete the virus without permission of user. This software is supported in android mobile phones. It has restore point method for replacing the deleted files.

\section{CONCLUSION}

Most of the antivirus does not have all kind of features. Every antivirus was created for a specific protection from virus. But Spartans antivirus developed for protecting the system in many methods. It helps the users to protect the system from virus attacks. Users do not worry about the malicious activities in system because Spartans will defend against all unwanted activities.

\section{REFERENCES}

1. Jason N. Gaylord, Christian wenz. Professional ASP.net 4.5 C\# and VB, Publisher :APRESS

2. Smadav (antivirus)-Graphical user interface

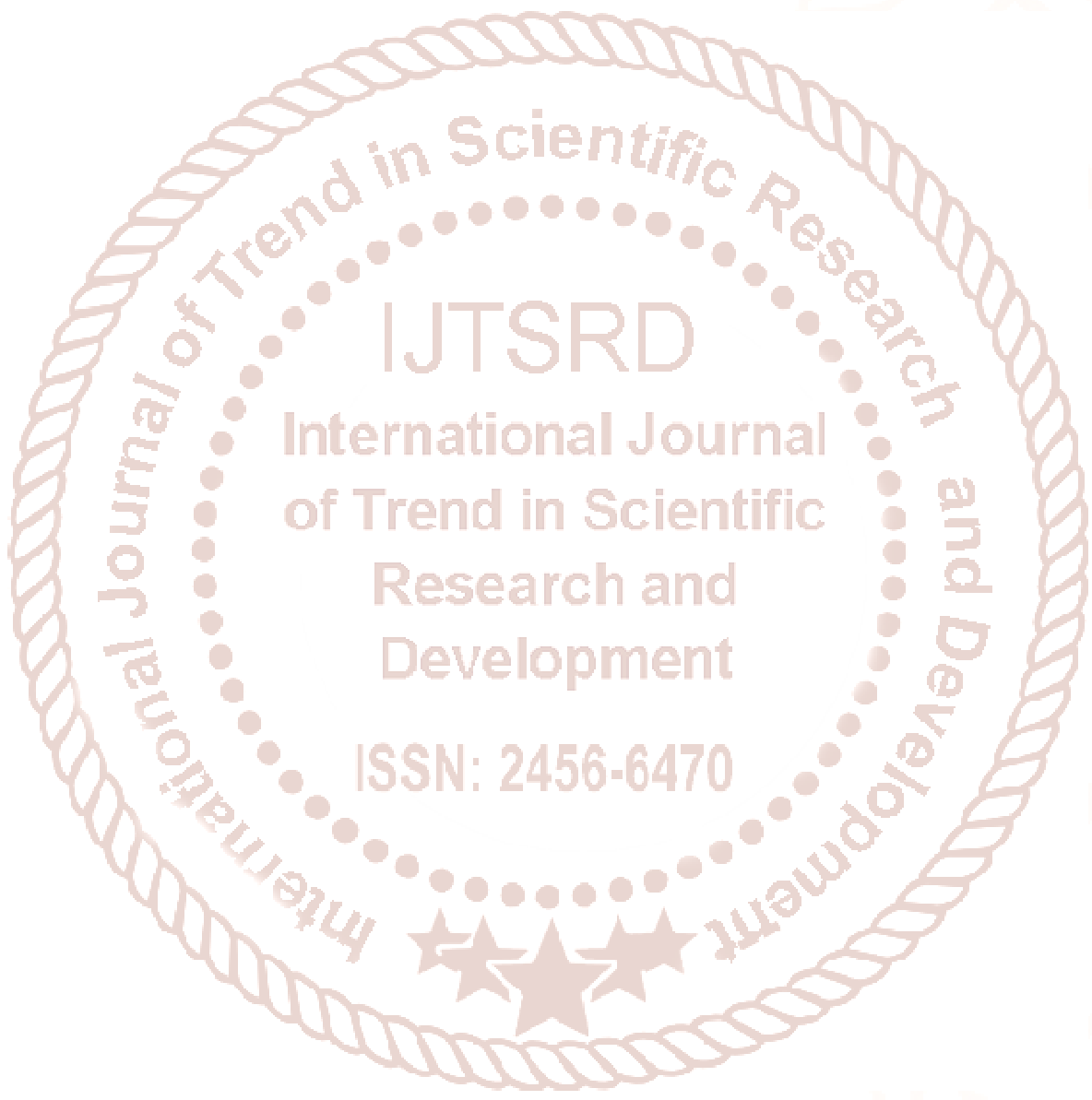

